\title{
Immunohistochemical Study of a Patient with Diffuse Pulmonary Corpora Amylacea Detected by Open Lung Biopsy
}

\author{
Hideo Yamanouchi, Takeo Yoshinouchi*, Ryohei Watanabe*, Jiro Fujita**, \\ Jiro TAKAHARA** and Yuji OHTSUKI
}

\begin{abstract}
The chest radiographs of an asymptomatic 58-year-old Japanese man with pulmonary corpora amylacea (PCA) revealed bilateral patchy and nodular infiltrates. Lung specimens obtained by open lung biopsy were histochemically and immunohistochemically analyzed. In all sections of dissected lung tissue, hematoxylin and eosin staining revealed homogeneous eosinophilic acellular round bodies (50-100 $\mu \mathrm{m}$ in diameter) containing granular black pigments in the alveolar spaces. Some round bodies were surrounded and phagocytized by alveolar macrophages. The laminated round bodies stained positively with PAS and Congo red. In addition, many of the rounded bodies contained particles which stained positively with Berlin blue. Immunohistochemical staining for epithelial membrane antigen (EMA) as well as PE-10 was distinctively positive. This is a very rare case of diffuse PCA found by open lung biopsy. Immunohistochemical examination suggested that PCA consisted of pulmonary surfactant protein and epithelial membrane antigen. (Internal Medicine 38: 900-903, 1999)
\end{abstract}

Key words: epithelial membrane antigen, surfactant apoprotein A, pulmonary corpora amylacea

\section{Introduction}

Pulmonary corpora amylacea (PCA), a round and laminated material which is stained by both eosin and Congo red, was first described by Friedreich in 1856 (1). PCA are incidentally found in the alveolar spaces of the lungs, particularly in the elderly (2). PCA are also reportedly observed in the pathological findings of certain degenerative and metabolic disorders such as, pneumonia, pulmonary infarction, pulmonary collapse, chronic heart failure, and other conditions (3). However, no general consensus concerning either the histogenesis or morphogenesis of PCA has been obtained (3).

Recently, we examined a 58-year-old man with diffuse PCA as detected by open lung biopsy. Here, the microscopic and immunohistochemical findings of PCA are described.

\section{Case Report}

A 58-year-old healthy Japanese man, a non-smoker, was admitted to Matsuyama-Shimin Hospital on April 12, 1995 due to an abnormal shadow on chest radiograph on a regular health examination. Although the same chest X-ray findings had been observed in 1994, no further examinations had been performed.
Physical examination revealed no abnormal findings. The hemogram, blood biochemical findings, immunological findings, blood gas analysis, and pulmonary function test results were all normal. His chest radiograph revealed bilateral patchy and nodular infiltrates (Fig. 1). High-resolution chest CT scan revealed abnormal patchy high density areas in both lungs (Fig. 2). On Ga scintigraphy, there was slightly abnormal uptake in both lungs. There was no mediastinal lymphadenopathy. Bronchoalveolar lavage fluid (BALF) analysis showed no specific abnormal finding, revealing cellular sediments of $81 \%$ macrophages, $11 \%$ lymphocytes, and $2 \%$ neutrophils. No malignant cells were observed on cytological examination of bronchoalveolar lavage fluid and smeared sputa. A lung specimen obtained by transbronchial biopsy demonstrated homogeneous or laminated round bodies in alveolar spaces. For further detailed examination, open lung biopsy was performed from the upper $\left(\mathrm{S}^{4}-\mathrm{S}^{5}\right)$ and lower $\left(\mathrm{S}^{9}\right)$ lobes of the left lung. Macroscopically, pleural fibrous adhesion was found in mediastinal and anterior aspects of the left lung, exhibiting incomplete lobulation of upper and lower lobes. The surface itself, and the cut surface of resected specimens showed no particular findings.

The dissected lung tissues $1.4 \times 1.0 \times 0.9 \mathrm{~cm}$ from $S^{9}$ and

From the Second Department of Pathology, Kochi Medical School, Kochi, *the Department of Internal Medicine and Surgery, Matsuyama-Shimin Hospital, Ehime and **the First Department of Internal Medicine, Kagawa Medical University, Kagawa

Received for publication October 14, 1998; Accepted for publication June 24, 1999

Reprint requests should be addressed to Dr. Jiro Fujita, the First Department of Internal Medicine, Kagawa Medical University, 1750-1, Miki-cho, Kita-gun, Kagawa 761-0793 


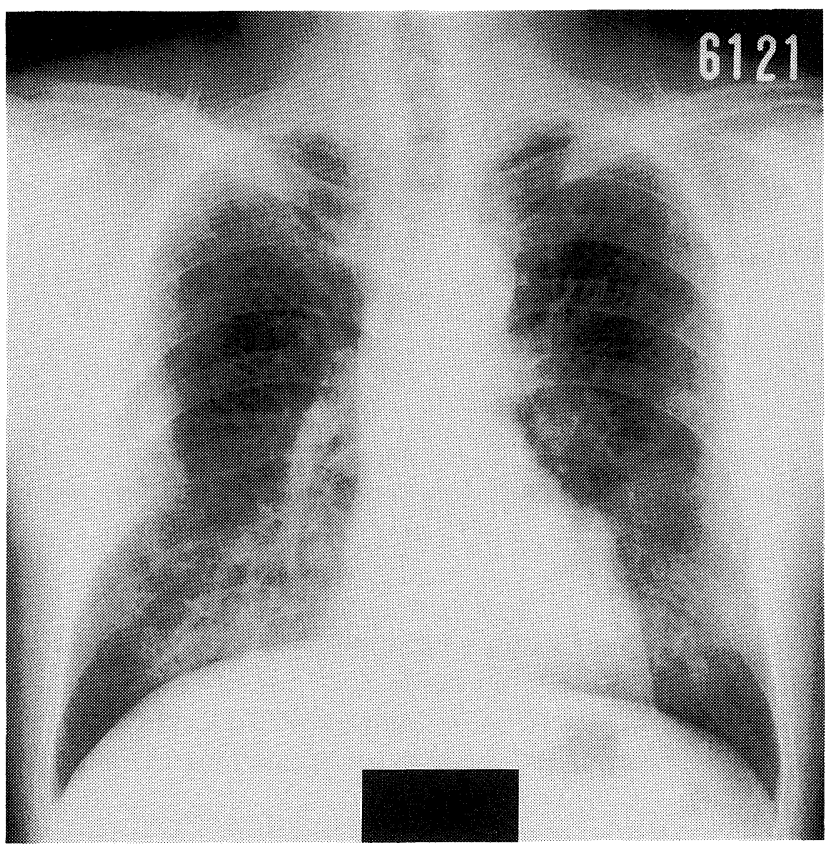

Figure 1. Chest radiograph obtained on admission, showing infiltrative shadows from middle to lower fields in both lungs.

$2.2 \times 1.0 \times 0.9 \mathrm{~cm}$ from $\mathrm{S}^{4}-\mathrm{S}^{5}$ were then histochemically and immunohistochemically evaluated. Routine hematoxylin and eosin (HE) staining as well as special staining, such as periodic acid-Schiff without or with diastase predigestion (PAS or D-PAS), mucicarmine, Congo red, Alcian blue, Berlin blue, Phosphotungstic acid hematoxylin (PTAH), Azan, silver impregnation, Elastica van Gieson (EVG), Giemsa, and modified methenamine silver-nitrate (Grocott) were performed. Immunohistochemical staining using the streptavidin-biotin-peroxidase method was performed using antibodies against the epithelial membrane antigen (EMA, 1:50, DAKO), surfactant apoprotein A (PE-10, 1:100, DAKO), S-100 (1:800, DAKO), and macrophages (HAM56, 1:4000, ENZO).

The results of histochemical and immunohistochemical staining are presented in Table 1. In all sections of dissected lung tissue $\left(S^{9}\right.$ and $\left.S^{4}-S^{5}\right)$, HE staining revealed homogeneous eosinophilic acellular round bodies, generally 50-100 micrometers in diameter, some contained granular black pigments in the alveolar spaces frequently in clusters (Fig. 3). The round bodies were laminated concentrically and were frequently surrounded by alveolar macrophages. In some areas, the small round bodies had been phagocytized by macrophages. The laminated round bodies stained positively with PAS, D-PAS, mucicarmine, and Congo red. In addition, many of the round bodies contained particles which stained positively with Berlin blue, in addition to the black granules. A few bodies were partially positive on PTAH staining. Other than these bodies, no particular findings were detected in the resected specimen.

Immunohistochemical staining for EMA was distinctly positive, revealing a concentric, linear laminated structure (Fig. 4).

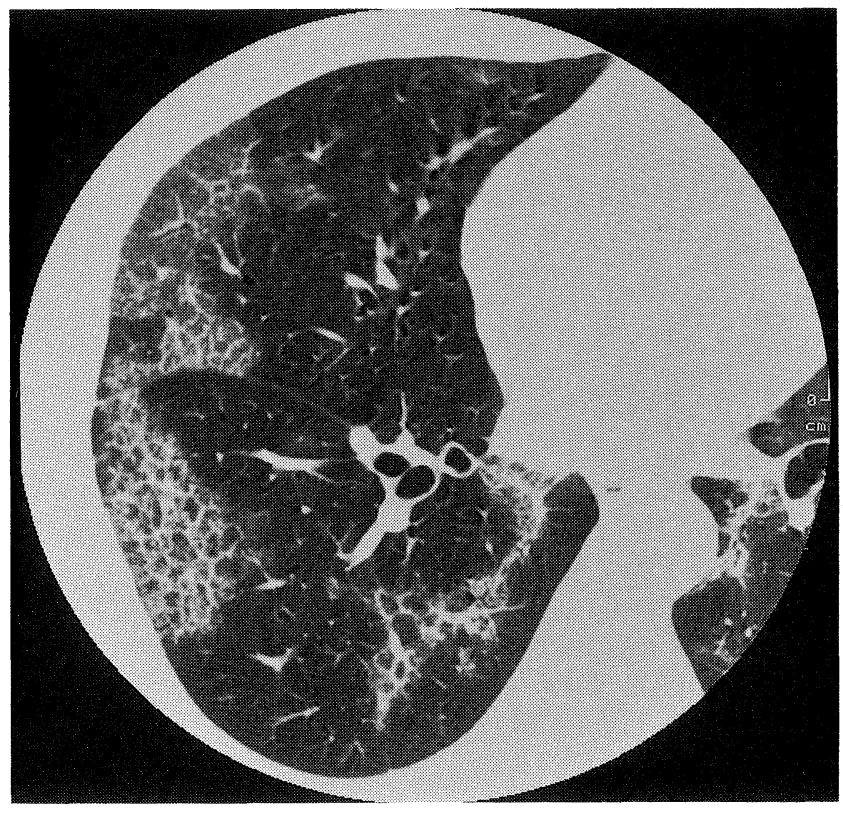

Figure 2. High-resolution chest CT scan on admission, showing bilateral patchy groundglass-like shadows in the lung fields.

Table 1. Histochemical and Immunohistochemical Characterization of Pulmonary Corpora Amylacea

\begin{tabular}{|c|c|}
\hline Stainings or reaction & Results \\
\hline PAS $^{1)}$ & + \\
\hline D-PAS ${ }^{1)}$ & + \\
\hline Mucicarmine & + \\
\hline Congo-red & $(+)$ \\
\hline Alcian blue & - \\
\hline Berlin blue & + \\
\hline $\mathrm{PTAH}^{2)}$ & $(+)$ \\
\hline Azan & - \\
\hline Silver impregnation & - \\
\hline $\mathrm{EVG}^{3)}$ & - \\
\hline Giemsa & - \\
\hline Grocott & - \\
\hline \multicolumn{2}{|l|}{ Antibodies examined } \\
\hline $\mathrm{EMA}^{4)}$ & + \\
\hline PE- $10^{5)}$ & + \\
\hline$S-100$ & - \\
\hline${\left.\text { HAM } 56^{6}\right)}^{2}$ & + \\
\hline
\end{tabular}

+: positive, (+): partially positive, - : negative. 1) PAS, D-PAS: Periodic acid-Shiff reaction without or with diastase predigestion, ${ }^{2)}$ PTAH: phosphotungstic acid hematoxylin, ${ }^{3)}$ EVG: elastica van Gieson, ${ }^{4)}$ EMA: epithelial membrane antigen, ${ }^{5)} \mathrm{PE}-10$ : antibody to human surfactant apoprotein $\mathrm{A},{ }^{6}$ ) HAM56: antibody to human alveolar macrophage. 


\section{YAMANOUCHI et al}

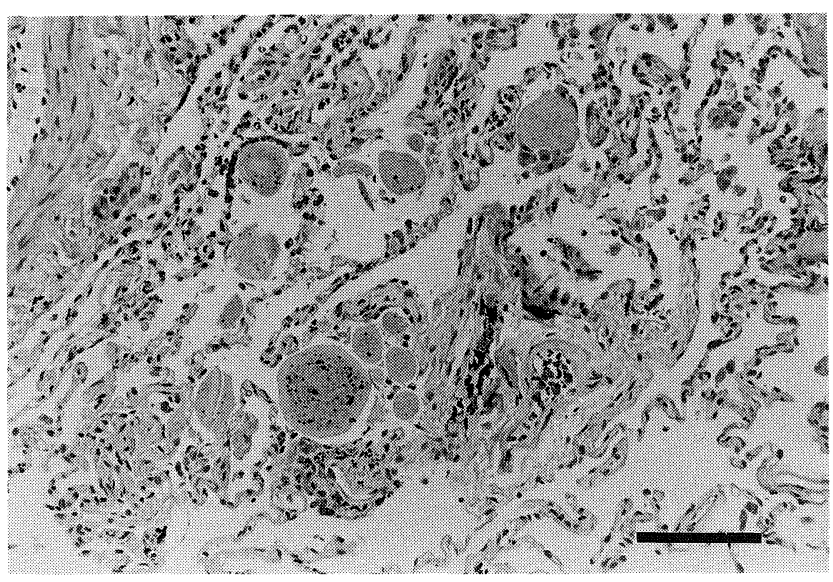

Figure 3. A general view of PCA observed in the alveolar spaces. Granular black deposits are observed in some PCA. AIveolar macrophages surround PCA (HE stain, $\times 180$, bar: 100 $\mu \mathrm{m})$. PCA: pulmonary corpora amylacea.

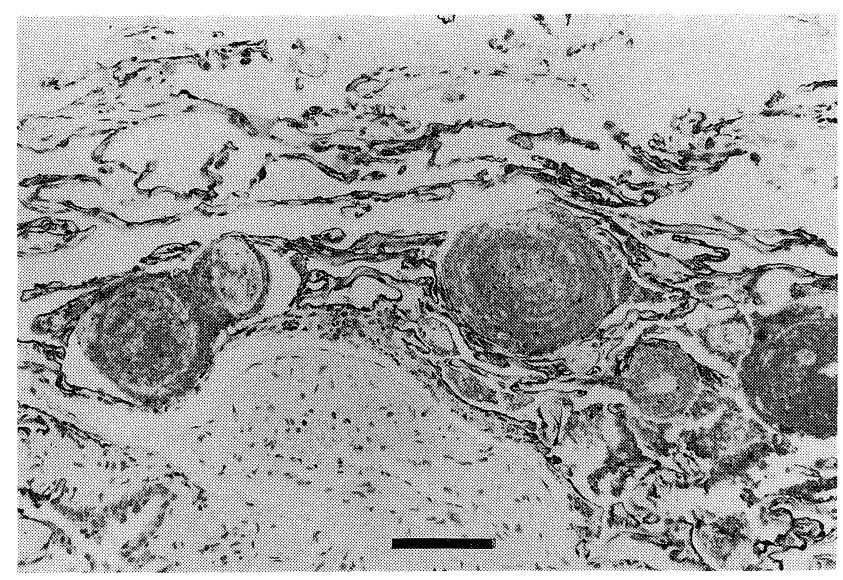

Figure 4. Immunohistochemical staining for EMA shows distinct positivity in PCA, revealing concentrically laminated structures. ABC method, $\times \mathbf{2 8 0}$ (bar: $50 \mu \mathrm{m}$ ). ABC: Streptavidin biotin peroxidase complex, EMA: epithelial membrane antigen, PCA: pulmonary corpora amylacea.

Immunohistochemical staining for PE-10, an antibody to human pulmonary surfactant $36 \mathrm{kD}$ apoprotein was also clearly and homogeneously positive (Fig. 5). Staining for HAM56 was diffusely positive, but that for $\mathrm{S}-100$ protein was negative.

\section{Discussion}

Although PCA as a pathological finding is well described in the pathology literature, a case of diffuse PCA detected by open lung biopsy is extremely rare.

PCA are acellular round bodies (approximately $60-100 \mu \mathrm{m}$ in diameter) with homogeneous or laminated structures usually found free in the alveolar spaces. PCA were first described

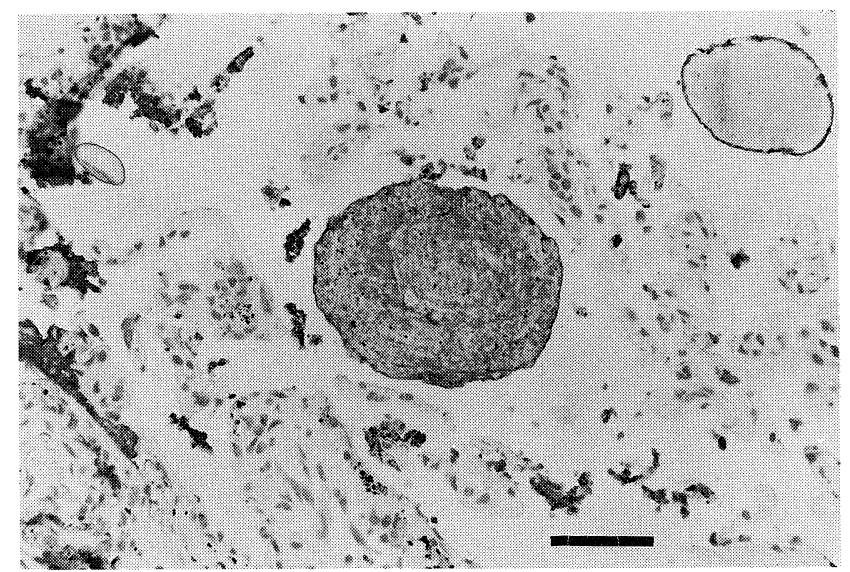

Figure 5. Immunohistochemical staining for PE10 is strongly positive in PCA. ABC method, $\times 280$ (bar: $50 \mu \mathrm{m}$ ). ABC: Streptavidin biotin peroxidase complex, PCA: pulmonary corpora amylacea, PE10: antibody to surfactant apoprotein $A$.

by Friedreich in 1856 (1). In 1957, Michaels and Levene reported that PCA are found in 41 out of 1,070 post-mortem lung sections in increasing frequency with advancing age (2). In addition, Hollander and Hutchins reported that PCA are found in 37 out of $6,500(0.6 \%)$ unselected autopsies (4). Spencer described that PCA are related to degenerative and metabolic disorders, pneumonia, pulmonary infarction, pulmonary collapse, or chronic heart failure (3).

The causes of PCA are still unclear. Dobashi et al evaluated PCA by immunohistochemical and electron microscopic analysis and suggested that PCA may be formed by sequential aggregation, fusion, and coalescence of degenerated alveolar macrophages (5). In the present case, alveolar macrophages including multinucleated macrophages surrounded PCA and phagocytized PCA. These findings suggest that the collection of macrophages might have been the result of reactions to foreign bodies. Hollander and Hutchins noted that the central particles may be of special importance, since particle-like foreign material may act as a nidus or nucleation center for the formation of PCA (4).

Baar and Ferguson studied the biochemical composition of PCA and concluded that glycoproteins are their principal constituents (6). The positive staining of PCA for PAS and D-PAS in a previous study (7) and the present study is consistent with this conclusion. Akino et al suggested that the glycoproteins included in PCA are pulmonary surfactant $36 \mathrm{kD}$ apoproteins (7). The present findings of staining with PE-10 also demonstrated that PCA contains the pulmonary surfactant apoprotein. In addition, staining with an antibody to EMA revealed a clear concentric structure in PCA, suggesting that EMA is also an important constitute for PCA. Additionally, it is of interest that PCA in this case contained Berlin blue-positive granules, appearing black in HE stain, although this patient was a nonsmoker.

In conclusion, we report a very rare case of diffuse PCA 


\section{Pulmonary Corpora Amylacea}

which was observed by open lung biopsy. Immunohistochemical examination demonstrated that pulmonary surfactant protein as well as epithelial membrane antigen are constituents of PCA.

\section{References}

1) Friedreich N. Kleinere Mittheilungen: I. Corpora amylacea in den Lungen. Virchows Archiv (Path Anat) 9: 613-618, 1856.

2) Michaels L, Levene C. Pulmonary corpora amylacea. J Pathol Bacteriol 74: 49-56, 1957.
3) Spencer H. Degenerative and metabolic disorders of the lungs. In: Pathology of the Lung (Third Edition). Vol. 2. Pergamon Press Ltd. New York, 1977: 680-681.

4) Hollander DH, Hutchins GM. Central spherules in pulmonary corpora amylacea. Arch Pathol Lab Med 102: 629-630, 1978.

5) Dobashi M, Yuda F, Narabayashi M, et al. Histopathological study of corpora amylacea pulmonum. Histol Histopathol 4: 153-165, 1989.

6) Baar HS, Ferguson FF. Microlithiasis alveolaris pulmonum. Association with diffuse interstitial pulmonary fibrosis. Arch Pathol 76: 659-666, 1963.

7) Akino T, Mizumoto M, Shimizu H, et al. Pulmonary corpora amylacea contain surfactant apoprotein. Pathol Res Pract 186: 687-691, 1990. 